\title{
Effect of magnetic field on peristaltic flow of Walters -B fluid through a porous medium in a tapered asymmetric channel.
}

\author{
Ahmed M.Abdulhadi ${ }^{1}$, Tamara S. Ahmed ${ }^{2}$ \\ College of science ${ }^{1}$, university of Baghdad \\ ahm6161@yahoo.com, \\ Collage of education for pure science (ibn al Haitham),university of Baghdad \\ Tamaraalshareef@yahoo.com
}

\begin{abstract}
The problem of peristaltic transport of an incompressible non-Newtonian fluid in a tapered a symmetric channel through a porous medium is presented under long-wave length and low -Reynolds number assumptions, the fluid is considered to be Walters - B fluid and electrically conducting by a transverse magnetic field. The tapered asymmetric channel in the flow induced by talking peristaltic wave imposed on the non-uniform boundary walls to possess different amplitudes and phases. Series solutions for stream function, axial velocity and pressure gradient are given using regular perturbation technique. Numerical computations have been performed for the pressure rise per wave length. The effect of the physical

parameters of the problem on these distributions are discussed and illustrated graphically through a set of figure
\end{abstract}

\section{Indexing terms/Keywords}

Walters-B fluid; Peristaltic transport; Magnetic field; Porous medium; Tapered a symmetric channel.

\section{Academic Discipline And Sub-Disciplines}

Mathematics, Physics, computer science, fluid mechanics in applied mathematics

\section{TYPE (METHOD/APPROACH)}

Applied Mathematics / fluid mechanics

\section{INTRODUCTION}

Peristaltic transport is a form of material transport included by a progressive wave of contraction or expansion along the length of distensible tube mixing and transporting the fluid in the direction of the wave propagation. This kind of phenomenon is termed as peristaltic. It plays an indispensable role in transporting many physiological fluids in the body under various situations as urine transport from kidney to bladder, the movement of chyme in the gastrointestinal tracts, transport of spermatozoa in the ductus efferent's of the male reproductive tract, movement of ovum in the fallopian tubes, swallowing of food through esophagus and the vasomation of small blood vessels many modern mechanical devices have been designed on the principle of peristaltic pumping to transport the fluids without internal moving parts, for example the blood pump in the heart-lung machine and peristaltic transport of naxious fluid in nuclear industry. The mechanism of peristaltic transport has attracted the attention of many investigators since its investigation by Latham [13], Burns and pareks[2], shapero et all.[22], Fung and yih [3], Takabatake and Ayukawa [25], Akram and Nedeem [1], mekheimer and Elkot [16], Mekheimor and al -arabi[15], mekheimer[14], Nadeamand akbar [19], Kothandapaniet al.[10], of peristaltic flow for different fluids have been reported under various conditions with reference to physiological and mechanical situations. Most of these investigations are confined to the peristaltic flow only in a symmetric channel or tube . consideration of wall properties in peristalsis is of special value in study of blood flow in arteries and veins, urine flow in the urethras and air flow in the lungs. Peristaltic motion in a complaint wall channel has also been investigated by some researchers. Radhakrishnamacharya and Srinivasulu [20] analyzed the influence of wall properties on peristaltic motion of Newtonian fluid with heat transfer. Peristaltic motion of micro polar fluid in circular cylindrical tubes with wall properties is discussed by muth et al [17], Hayat et al.[5, 6] examined the MHD peristaltic flow of Jeffery and Johnson -Segalman fluids with compliant walls.srinivas and kothandapani [23] analyzed the heat and mass transfer effects on MHD peristaltic flow of Newtonian fluid in a porous channel with compliant walls. Riaz et al.[21] investigated the peristaltic motion of prandtl fluid in rectangular duct with wall properties. Recently, peristaltic flow of burgers fluid in complaint walls channel was investigated by javed et al.[9]. Peristaltic flow with complaint walls and hall current was studied by gad [4]. Very recently, the combined influence of heat and mass transfer on the peristaltic motion of pseudeplastic fluid with wall properties was analytically explored by hina et al.[7]. Slip effects on the peristaltic flow of eyring- powell fluid with wall properties were examined by hina [8]. Amongst the many suggested models, walters [26] has developed a physically accurate 
mathematical model for the rhedogical equation of state of a viscoelastic fluid with short memory. This model has been Shawn to capture the characteristic of actual viscoelastic polymer solutions, hydrocarbons, paints and other chemical engineering fluids. The walters-B fluid model generates highly non-linear flow equations which have order higher than that of the Navier-stokes equations. It also in eorporates elastic properties of the fluid which are important in extensional behavior of polymers. Peristalsis of Walters-B fluid with wall properties has never been addressed previously, Thus margiam javed et al.[18] is undertaking to fill this void by incorporating velocity slip and temperature jump conditions. In the present paper, we investigated the peristaltic transport of walters-B fluid under the effect of magnetic field through a porous medium in a tapered a symmetric channel. Regular perturbation technique are used under long-wave length (wave number is small ) and law-Rynolds assumptions. Series solutions for stream function, axial velocity and pressure gradient are given, numerical computations have been performed for pressure rise per wave length. The influence of the physical parameters of the problem are discussed and illustrated graphically.

\section{MATHEMATICAL MODELS}

Let us consider the MHD flow of an incompressible and electrically conducting walters -B fluid through a porous medium of two -dimensional tapered a symmetric channel. We assume that infinite wave train traveling with velocity $\mathrm{C}$ along the non -uniform walls. We choose a rectangular coordinate system for the channel with $\bar{X}$ along the direction of wave propagation and parallel to the centre line and $\bar{Y}$ transverse to it. The wall of the tapered a symmetric channel are given in fig.(1) by the equations

$$
\begin{aligned}
& \overline{H_{1}}(\bar{x}, \bar{t})=-d-m^{\prime} \bar{x}-a_{1} \sin \left[\frac{2 \pi}{\lambda}(\bar{x}-c \bar{t})+\phi\right] \ldots . . \text { lower wall } \\
& \overline{H_{2}}(\bar{x}, \bar{t})=d+m^{\prime} \bar{x}+a_{2} \sin \left[\frac{2 \pi}{\lambda}(\bar{x}-c \bar{t})\right] \ldots . . . \text { upper wall }
\end{aligned}
$$

Where $a_{1}, a_{2}$ are the amplitudes of the waves, $\lambda$ is the wave length, $2 \mathrm{~d}$ is the width of the channel at the inlet, $m^{\prime}\left(m^{\prime}<<1\right)$ is the non-uniform parameters, the phase difference $\phi$ varies in the range $0 \leq \phi \leq \pi, \phi=0$ represents to symmetric channel in which waves are out of phase and when $\phi=\pi$ the waves are in phase, and further $a_{1}, a_{2}, d$ and $\phi$ satisfies the condition :

$$
a_{1}^{2}+a_{2}^{2}+2 a_{1} a_{2} \cos \phi \leq(2 d)^{2}
$$

The constitutive equations for Walters-B fluid are:

$$
\begin{aligned}
& S=-P I+\varsigma, \\
& \varsigma=2 \eta_{0} e-2 k_{0} \frac{\overline{\delta e}}{\overline{\delta t}}, \\
& e=\nabla v+(\nabla v)^{T}, \\
& \overline{\frac{\delta e}{\overline{\delta t}}}=\frac{\partial e}{\partial t}+v \cdot \nabla e-e \nabla v-(\nabla v)^{T} e,
\end{aligned}
$$

In which $\mathrm{S}$ is the Cauchy stress tensor, $-\mathrm{PI}$ is the spherical part of the stress due to constrain of in compressibility, $\delta$ is the extra stress tensor, $\eta_{0}$ is the coefficient of viscosity, e is the rate of strain tensor and $\frac{\bar{\delta}}{\overline{\delta t}}$ denotes the convicted differentiation of a tensor quantity in relation to the material motion 


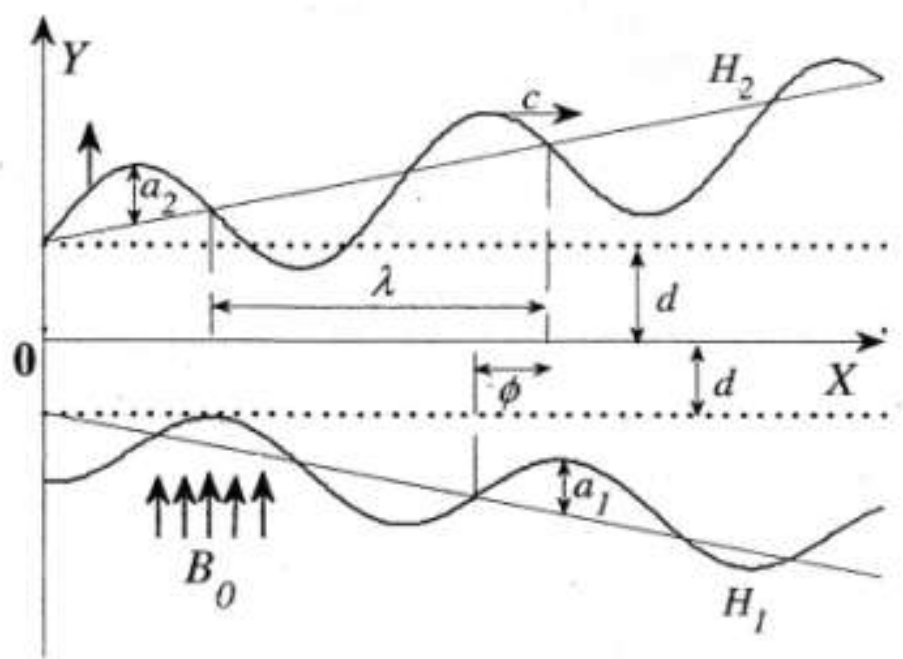

Fig. 1 A physical sketch of the problem.

The governing equations of the continuity and momentum equations for two-dimensional case are:

$$
\begin{aligned}
& \frac{\partial \bar{U}}{\partial \bar{X}}+\frac{\partial \bar{V}}{\partial \bar{Y}}=0 \\
& \rho\left(\frac{\partial \bar{U}}{\partial t}+\bar{U} \frac{\partial \bar{U}}{\partial \bar{X}}+\bar{V} \frac{\partial \bar{U}}{\partial \bar{Y}}\right)=-\frac{\partial \bar{P}}{\partial \bar{X}}+\frac{\partial}{\partial \bar{X}}(\bar{S} \overline{X X})+\frac{\partial}{\partial \bar{Y}}(\bar{S} \overline{X Y})-\sigma B_{0}^{2} \bar{U}-\frac{\eta_{0}}{k} \bar{U} \\
& \rho\left(\frac{\partial \bar{V}}{\partial \bar{t}}+\bar{U} \frac{\partial \bar{V}}{\partial \bar{X}}+\bar{V} \frac{\partial \bar{V}}{\partial \bar{Y}}\right)=-\frac{\partial \bar{P}}{\partial \bar{Y}}+\frac{\partial}{\partial \bar{X}}(\bar{S} \overline{X Y})+\frac{\partial}{\partial \bar{Y}}(\bar{S} \overline{Y Y})-\frac{\eta_{0}}{k} \bar{V}
\end{aligned}
$$

where the stress components are given by

$$
\begin{aligned}
& \bar{S} \overline{x x}=4 \eta_{0} \frac{\partial \bar{U}}{\partial \bar{X}}-2 k_{0}\left[2 \frac{\partial^{2} \bar{U}}{\partial \bar{X} \partial t}+2\left(\bar{U} \frac{\partial^{2} \bar{U}}{\partial \bar{X}^{2}}+\bar{V} \frac{\partial^{2} \bar{U}}{\partial \bar{X} \partial \bar{Y}}\right)-4\left(\frac{\partial \bar{U}}{\partial \bar{X}}\right)^{2}-2 \frac{\partial \bar{V}}{\partial \bar{X}}\left(\frac{\partial \bar{U}}{\partial \bar{Y}}+\frac{\partial \bar{V}}{\partial \bar{X}}\right)\right] \\
& \bar{S} \overline{X Y}=2 \eta_{0}\left(\frac{\partial \bar{U}}{\partial \bar{Y}}+\frac{\partial \bar{V}}{\partial \bar{X}}\right)-2 k_{0}\left[\frac{\partial^{2} \bar{U}}{\partial \bar{Y} \partial \bar{t}}+\frac{\partial^{2} \bar{V}}{\partial \bar{X} \partial t}-2 \frac{\partial \bar{U}}{\partial \bar{X}} \frac{\partial \bar{U}}{\partial \bar{Y}}+\left(\bar{U} \frac{\partial}{\partial \bar{X}}+\bar{V} \frac{\partial}{\partial \bar{Y}}\right)\left(\frac{\partial \bar{U}}{\partial \bar{Y}}+\frac{\partial \bar{V}}{\partial \bar{X}}\right)\right. \\
& \left.-\frac{\partial \bar{V}}{\partial \bar{Y}}\left(\frac{\partial \bar{U}}{\partial \bar{Y}}+\frac{\partial \bar{V}}{\partial \bar{X}}\right)-\frac{\partial \bar{U}}{\partial \bar{X}}\left(\frac{\partial \bar{U}}{\partial \bar{Y}}+\frac{\partial \bar{V}}{\partial \bar{X}}\right)-2 \frac{\partial \bar{V}}{\partial \bar{X}} \frac{\partial \bar{V}}{\partial \bar{Y}}\right] \\
& \bar{S} \overline{Y Y}=4 \eta_{0} \frac{\partial \bar{V}}{\partial \bar{Y}}-2 k_{0}\left[2 \frac{\partial^{2} \bar{V}}{\partial \bar{Y} \partial t}+2\left(\bar{U} \frac{\partial^{2} \bar{V}}{\partial \bar{X} \partial \bar{Y}}+\bar{V} \frac{\partial^{2} \bar{V}}{\partial \bar{Y}^{2}}\right)-4\left(\frac{\partial \bar{V}}{\partial \bar{Y}}\right)^{2}-2 \frac{\partial \bar{U}}{\partial \bar{Y}}\left(\frac{\partial \bar{U}}{\partial \bar{Y}}+\frac{\partial \bar{V}}{\partial \bar{X}}\right)\right]
\end{aligned}
$$

in which $\rho$ is the fluid density ,P is the pressure, $(\bar{U}, \bar{V})$ are velocity components in the direction of the laboratory frame $(\bar{X}, \bar{Y}), \sigma$ is the electrically conductivity and $\mathrm{B}_{0}$ is the constant magnetic field.

We employ the following dimensionless variables in the governing equations of motion: 
$x=\frac{\bar{X}}{\lambda}, y=\frac{\bar{Y}}{d}, t=\frac{c \bar{t}}{\lambda}, u=\frac{\bar{U}}{c}, v=\frac{\bar{V}}{\delta c}, h_{1}=\frac{\overline{H_{1}}}{d}, h_{2}=\frac{\overline{H_{2}}}{d}$

$\rho=\frac{d^{2} \bar{P}}{c \lambda \eta_{0}}, a=\frac{a_{1}}{d}, b=\frac{a_{2}}{d}, m=\frac{m^{\prime} \lambda}{d}, S=\frac{d}{\eta_{0} c} \bar{S}, \delta=\frac{d}{\lambda}$

$M=\sqrt{\frac{\sigma}{\eta_{0}}} B_{0} d, \operatorname{Re}=\frac{\rho e d}{\eta_{0}}, \bar{k}=\frac{k_{0} c}{\eta_{0} d}, k^{2}=\frac{1}{D r}=\frac{d^{2}}{k}$

The stream function $\psi(x, y)$ is defined by $: u=\frac{\partial \psi}{\partial y}, v=\frac{-\partial \psi}{\partial x}$

Eq.(7) is satisfied automatically and Eqs.(8),(9),(10),(11) and (12) become:

$\operatorname{Re} \delta\left(\psi_{y t}+\psi_{y} \psi_{x y}-\psi_{x} \psi_{y y}\right)=-\frac{\partial p}{\partial x}+\delta S_{x x x}+S_{x y y}-\left(M^{2}+k^{2}\right) \psi_{y}$

$\operatorname{Re} \delta^{3}\left(\psi_{x t}-\psi_{y} \psi_{x x}+\psi_{x} \psi_{x y}\right)=-\frac{\partial p}{\partial y}+\delta^{2} S_{x y x}+\delta S_{x y y}+\delta^{2} k^{2} \psi_{x}$

$S_{x x}=4 \delta \psi_{x y}-2 \bar{k} \delta^{2}\left[2 \psi_{x y t}+2\left(\psi_{y} \psi_{x x y}-\psi_{x} \psi_{x y y}\right)-4 \psi_{x y}^{2}+2 \psi_{x x}\left(\psi_{y y}-\delta^{2} \psi_{x x}\right)\right]$

$S_{x y}=2\left(\psi_{y y}-\delta^{2} \psi_{x x}\right)-2 \bar{k}\left[\delta \psi_{y y t}-\delta^{3} \psi_{x x t}+\delta \psi_{y} \psi_{x y y}-\delta^{3} \psi_{y} \psi_{x x x}-\delta \psi_{x} \psi_{y y y}+\right.$

$\left.\delta^{3} \psi_{x} \psi_{x x y}-2 \delta \psi_{x y} \psi_{y y}-2 \delta^{3} \psi_{x x} \psi_{x y}\right]$

$S_{y y}=-4 \delta \psi_{x y}-2 \bar{k}\left[\delta^{2}\left(-2 \psi_{x y t}-2\left(\psi_{y} \psi_{x x y}+2 \psi_{x x} \psi_{y y}-4 \psi_{x y}^{2}-2 \psi_{y y}^{2}\right]\right.\right.$

Now, in the laboratory frame $(\bar{X}, \bar{Y})$ the flow is unsteady, However if treated it as steady flow in the wave frame $(\mathrm{x}, \mathrm{y})$, so the equations (13), (14), (15), (16) and (17) can be written as:

$\operatorname{Re} \delta\left(\psi_{y} \psi_{x y}-\psi_{x} \psi_{y y}\right)=-\frac{\partial p}{\partial x}+\delta S_{x x x}+S_{x y y}-N_{1}^{2} \psi_{y}$

$\operatorname{Re} \delta^{3}\left(-\psi_{y} \psi_{x x}+\psi_{x} \psi_{x y}\right)=-\frac{\partial p}{\partial y}+\delta^{2} S_{x y x}+\delta S_{y y y}+\delta^{2} k^{2} \psi_{x}$

$S_{x x}=4 \delta \psi_{x y}-2 \bar{k} \delta^{2}\left[2\left(\psi_{y} \psi_{x x y}-\psi_{x} \psi_{x y y}\right)-4 \psi_{x y}^{2}+2 \psi_{x x}\left(\psi_{y y}-\delta^{2} \psi_{x x}\right)\right]$

$S_{x y}=2\left(\psi_{y y}-\delta^{2} \psi_{x x}\right)-2 \bar{k}\left[\delta \psi_{y} \psi_{x y y}-\delta^{3} \psi_{y} \psi_{x x x}-\delta \psi_{x} \psi_{y y y}+\right.$

$\left.\delta^{3} \psi_{x} \psi_{x x y}-2 \delta \psi_{x y} \psi_{y y}-2 \delta^{3} \psi_{x x} \psi_{x y}\right]$

$S_{y y}=-4 \delta \psi_{x y}-2 \bar{k}\left[\delta^{2}\left(-2 \psi_{y} \psi_{x x y}+2 \psi_{x} \psi_{x y y}+2 \psi_{x x} \psi_{y y}-4 \psi_{x y}^{2}\right)-2 \psi_{y y}^{2}\right]$

Where $N_{1}^{2}=\left(M^{2}+k^{2}\right) ; \delta$ is the wave number, $\mathrm{M}$ is the Hartmann number, Re is the Reynolds number, $\bar{k}$ is kaba number, Dr is dercy number .

Under the long wave length and low Reynolds number approximations, equations (18) and (19) can be written as : 


$$
\begin{aligned}
& -\frac{\partial p}{\partial x}+S_{x y y}+S_{x y y}-N_{1}^{2} \psi_{y}=0 \\
& \frac{\partial p}{\partial y}=0
\end{aligned}
$$

equations (24) indicates that $P$ is the independent of $\mathrm{y}$, from equation (23)we have

$$
S_{x y y y}-N_{1}^{2} \psi_{y y}=0
$$

Which equivalent to

$\frac{\partial^{2}}{\partial y^{2}} S_{x y}-N_{1}^{2} \frac{\partial^{2} \psi}{\partial y^{2}}=0$

The appropriate boundary conditions in dimensions less form are :

$$
\begin{aligned}
& \psi=\frac{F}{2}, \frac{\partial \psi}{\partial y}=0, a t\left(y=h_{2}\right) \\
& \psi=\frac{-F}{2}, \frac{\partial \psi}{\partial y}=0, a t\left(y=h_{1}\right)
\end{aligned}
$$

We note that $h_{1}(x, t)$ and $h_{2}(x, t)$ represent the dimensionless form of the surfaces of peristaltic walls:

$$
\begin{aligned}
& h_{2}=1+m x+b \sin (2 \pi(x-t)) \text { and } \\
& h_{1}=-1-m x-a \sin (2 \pi(x-t)+\phi)
\end{aligned}
$$

\section{METHOD OF SOLUTION}

It is clear that the resulting equation of motion Eq.(26) is non linear. It seems to be impossible to obtain the general solution in closed form for orbiting values of all parameters a rising in this non linear equation. We seek the solution of the problem as a power series expansion in small parameter $\delta$. For perturbation solution, we expand $\psi, F, S_{x y}$ and $p$ as:

$$
\begin{aligned}
& \psi=\psi_{0}+\delta \psi_{1}+\ldots \ldots \ldots \ldots \\
& F=F_{0}+\delta F_{1}+\ldots \ldots \ldots \ldots \ldots \\
& S_{x y}=\left(S_{x y}\right)_{0}+\delta\left(S_{x y}\right)_{1}+\ldots \ldots \ldots . . \\
& p=p_{0}+\delta p_{1}+\ldots \ldots \ldots
\end{aligned}
$$

Substitution of above equations into Eq.(23) and (26) and boundary conditions Eq.(27) and collection of terms with respect to like powers of $\delta$ yields the following systems: 


\subsection{System of order zero :}

$2 \frac{\partial^{4} \psi_{0}}{\partial y^{4}}-N_{1}^{2} \frac{\partial^{2} \psi_{0}}{\partial y^{2}}=0$

$\frac{\partial p_{0}}{\partial x}=2 \frac{\partial^{3} \psi_{0}}{\partial y^{3}}-N_{1}^{2} \frac{\partial \psi_{0}}{\partial y}$

$\left(S_{x y}\right)_{0}=2 \frac{\partial^{2} \psi_{0}}{\partial y^{2}}$

$\psi_{0}=\frac{F_{0}}{2}, \frac{\partial \psi_{0}}{\partial y}=0, a t\left(y=h_{2}\right)$

$\psi_{0}=\frac{-F_{0}}{2}, \frac{\partial \psi_{0}}{\partial y}=0, a t\left(y=h_{1}\right)$

$2 \frac{\partial^{4} \psi_{1}}{\partial y^{4}}-N_{1}^{2} \frac{\partial^{2} \psi_{1}}{\partial y^{2}}=2 \bar{k} \frac{\partial^{2}}{\partial y^{2}}\left(\frac{\partial \psi_{0}}{\partial y} \frac{\partial^{3} \psi_{0}}{\partial x \partial^{2} y}-\frac{\partial \psi_{0}}{\partial x} \frac{\partial^{3} \psi_{0}}{\partial y^{3}}-2 \frac{\partial^{2} \psi_{0}}{\partial x \partial y} \frac{\partial^{2} \psi_{0}}{\partial y^{2}}\right)$

$\frac{\partial p_{1}}{\partial x}=\left(2 \frac{\partial^{3} \psi_{1}}{\partial y^{3}}-N_{1}^{2} \frac{\partial \psi_{1}}{\partial y}\right)-2 \bar{k} \frac{\partial}{\partial y}\left(\frac{\partial \psi_{0}}{\partial y} \frac{\partial^{3} \psi_{0}}{\partial x \partial y}-\frac{\partial \psi_{0}}{\partial x} \frac{\partial^{3} \psi_{0}}{\partial y^{3}}-2 \frac{\partial^{2} \psi_{0}}{\partial x \partial y} \frac{\partial^{2} \psi_{0}}{\partial y^{2}}\right)$

$\left(S_{x y}\right)_{1}=2 \frac{\partial^{2} \psi_{1}}{\partial y^{2}}-2 \bar{k}\left(\frac{\partial \psi_{0}}{\partial y} \frac{\partial^{3} \psi_{0}}{\partial x \partial y^{2}}-\frac{\partial \psi_{0}}{\partial x} \frac{\partial^{3} \psi_{0}}{\partial y^{3}}-2 \frac{\partial^{2} \psi_{0}}{\partial x \partial y} \frac{\partial^{2} \psi_{0}}{\partial y^{2}}\right)$

$\psi_{1}=\frac{F_{1}}{2}, \frac{\partial \psi_{1}}{\partial y}=0, a t\left(y=h_{2}\right)$

$\psi_{1}=\frac{-F_{1}}{2}, \frac{\partial \psi_{1}}{\partial y}=0, a t\left(y=h_{1}\right)$

\subsection{Solution for system of order $\left(\delta^{(0)}\right)$}

It is found that the solution of equation (30) under the associated boundary condition (33) is given by

$$
\begin{aligned}
& \quad \psi_{0}=n_{2} e^{n_{1} y} a_{1}+n_{2} e^{-n_{1} y} a_{2}+a_{3}+a_{4} y ; \\
& \text { where }\left(n_{1}=\frac{N_{1}}{\sqrt{2}}, n_{2}=\frac{2}{N_{1}^{2}}\right)
\end{aligned}
$$

$a_{1}, a_{2}, a_{3}, a_{4}$ are constants can be determinates by using the boundary conditions in Eq.(33).

In order to discuss the results quantitatively, we assume the instantaneous volume rate of the flow

t), (kothandapani and prakash [11], Kothandapani et al.[12], Srivastava et al,[24] ) as

$F(x, t)$, periodic in $(x-$

$$
F(x, t)=\theta+b \sin (2 \pi(x-t)+\phi)+a \sin (2 \pi(x-t))
$$

In which $\theta$ is the time-average of the flow over one period of the wave and 
$F=\int_{h_{1}}^{h_{2}} u d y$

$F_{0}=F-o\left(\delta F_{1}\right)$ and then neglecting the terms greater than $o(\delta)$

\subsection{Solution for system of $\operatorname{order}\left(\delta^{(1)}\right)$}

$$
\begin{aligned}
& \psi_{1}=\frac{e^{n_{1} y} c[1]}{n_{1}^{2}}+\frac{e^{-n_{1} y} c[2]}{n_{1}{ }^{2}}+c[3]+c[4] y+\left(e ^ { n _ { 1 } y } F _ { 0 } ^ { 2 } n _ { 1 } ^ { 2 } \left(2 e ^ { h _ { 1 } + h _ { 2 } ) n _ { 1 } } \left(-\left(h_{1}-h_{2}\right) h_{6} n_{1}\left(-5+2 n_{1} y\right)+\right.\right.\right. \\
& h_{4}\left(23-5 h_{5} n_{1}-6 n_{1} y+2 h_{5} n_{1}^{2} y-2 n_{1}^{2} y^{2}+h_{2} n_{1}\left(5-2 n_{1} y\right)+h_{1} n_{1}\left(-5+2 n_{1} y\right)\right)+ \\
& h_{3}\left(-23+5 h_{5} n_{1}+6 n_{1} y-2 h_{5} n_{1}^{2} y+2 n_{1}^{2} y^{2}+h_{2} n_{1}\left(5-2 n_{1} y\right)+h_{1} n_{1}\left(-5+2 n_{1} y\right)\right)+ \\
& e^{2 h_{1} n_{1}}\left(-h_{6}\left(-2+h_{1} n_{1}-h_{2} n_{1}\right)\left(-5+2 n_{1} y\right)+h_{4}\left(-13+2 n_{1} y+2 n_{1}^{2} y^{2}+h_{5} n_{1}\left(5-2 n_{1} y\right)\right)+\right. \\
& h_{3}\left(33-5 h_{5} n_{1}-10 n_{1} y+2 h_{5} n_{1}^{2} y+2 n_{1}^{2} y^{2}+2 h_{2} n_{1}\left(5-2 n_{1} y\right)+2 h_{1} n_{1}\left(-5+2 n_{1} y\right)\right)+ \\
& e^{2 h_{2} n_{1}}\left(-h_{6}\left(2+h_{1} n_{1}-h_{2} n_{1}\right)\left(-5+2 n_{1} y\right)+h_{4}\left(-33+5 h_{5} n_{1}+10 n_{1} y-2 h_{5} n_{1}^{2} y+2 n_{1}^{2} y^{2}+\right.\right. \\
& \frac{\left.\left.\left.2 h_{2} n_{1}\left(5-2 n_{1} y\right)+2 h_{1} n_{1}\left(-5+2 n_{1} y\right)\right)+h_{3}\left(13-2 n_{1} y-2 n_{1}^{2} y^{2}+h_{5} n_{1}\left(5-2 n_{1} y\right)\right)\right) \bar{k}\right)}{\left(8\left(e^{h_{1} n_{1}}\left(-2+h_{1} n_{1}-h_{2} n_{1}\right)+e^{2 h_{2} n_{1}}\left(2+h_{1} n_{1}-h_{2} n_{1}\right)\right)^{3}\right)+\ldots \ldots \ldots . .}
\end{aligned}
$$

where

$h_{3}=-m-2 a \pi \cos (2 \pi(x-t)+\phi)$;

$h_{4}=m+2 b \pi \cos (2 \pi(x-t))$;

$h_{5}=b \sin (2 \pi(x-t))-a \sin (2 \pi(x-t)+\phi)$;

$h_{6}=2 b \pi \cos (2 \pi(x-t))-2 a \pi \cos (2 \pi(x-t)+\phi)$;

$F_{0}=\theta+a \sin (2 \pi(x-t))+b \sin (2 \pi(x-t)+\phi) ;$

and $h_{1}, h_{2}$ determinate are given by Eq.(28) and c[1], c[2], c[3], c[4] are constants can be determinates by using the boundary conditions in Eq.(37). The non- dimensional expression for the average rise in pressure $\Delta p$ is given as follows:

$$
\Delta p=\int_{0}^{1} \int_{0}^{1} \frac{\partial p}{\partial x} d x d t
$$

\subsection{Pumping characteristics}

We plot the expression for $\Delta p$ in Eq.(42) against $\theta$ for various values of parameters of interest in Fig.(27). Numerical calculations for several values of Hartmann number $(\mathrm{M})$, the phase difference $(\phi)$, the non- uniform parameter of the channel $(\mathrm{m})$, the inverse of Darcy number $(\mathrm{K})$ and lower walls ( $a \& b$ )have been carried out. The effect of these parameters on $\Delta p$ have been evaluated numerically using mathematica and the results are presented graphically. In fig.(2), the effect of Hartmann number $\mathrm{M}$ on $\Delta p$ are seen, observed that in the pumping $\Delta p>0$ and the co-pumping ( $\Delta p<0$ )for the Walters-B fluid, an increase in $\mathrm{M}$ causes decreasing in the pumping increasing with an increase in $\mathrm{M}$. in Fig.(3), the effect of phase difference $\phi$ on $\Delta p$ are seen, observed that an increase in $\phi$ causes increasing in the co- 
pumping $(\Delta p<0)$ and decreasing in the pumping $\Delta p>0$. The effects of non-uniform parameter $\mathrm{m}$ as well as the amplitude of lower wall of channel a are plotted respectively in Figs.(4) and (5), it examined that an increase in $\mathrm{m}$ and a causes an increase in the pumping $\Delta p>0$ and decrease in the free pumping $\Delta p=0$ and co-pumping $\Delta p<0$. The effects of amplitude of upper wall of channel $\mathrm{b}$ and the inverse of Darcy number $\mathrm{K}$ on $\Delta p$ are plotted respectively in Figs.(6) and (7), it noticed that an increase in band $\mathrm{K}$ causes an increase in the pumping $\Delta p>0$ and free pumping $\Delta p=0$ and decrease in the co-pumping $\Delta p<0$.

\subsection{Velocity distribution}

Influences of geometric parameters on the velocity distribution have been illustrated in Fig.(8-14) these figures are scratched at the fixed values of $x=0.3, t=0.5$. the change in values of $m$ on the axial velocity $u$ is shown in fig.(8), it can be found that the axial velocity $u$ decrease with an increase in $m$ at the centre of channel and after $y=-0.5$ at the lower wall of the channel but after $y=+0.5$ at the upper wall of channel the velocity $u$ increase with an increase in $\mathrm{m}$. Fig.(9) shows the influence of $\phi$ on the axial velocity $u$, it observed that an increase in $\phi$ causes an increase in $u$ at the centre of channel and decreasing in $u$ after $y=-0.5$ at the lower part of channel.Fig.(10) displays the effect of a on the axial velocity $u$, it examined that an increase in a causes an increase in $u$ after $y=-0.5$ at the lower part of channel and decreasing in $u$ at the centre of the channel .Fig.(11) shows the effect of $b$ on the axial velocity $u$, it observed that an increase in $b$ causes decreasing in $u$ at the centre of channel and upper wall of channel. The influence of $M$ on the axial velocity $u$ is shown in Fig.(12), it noticed that an increase in $M$ causes decrease in $u$ at the centre of channel which is the same behavior of effect of $\mathrm{K}$ on axial velocity $\mathrm{u}$ and it is platted in Fig.(13) and conversely behavior of effect of $\theta$ on axial velocity $\mathrm{u}$ which is shown in Fig.(14).

\subsection{Trapping phenomenon}

The phenomenon of trapping is another interesting topic in peristaltic transport. The formation of an internally circulating bolus of fluid through closed stream lines is called trapping and this trapped bolus is pushed a head along with the peristaltic waves. The trapping for different values of $\mathrm{m}, \phi, \mathrm{a}, \mathrm{b}, \mathrm{M}, \mathrm{K}$ and $\theta$ are shown in Figs.(15-21) at fixed values of $(t=0.5)$. The stream lines for different values of $m$ are shown in Fig.(15), it has been noticed that the bolus increasing in the lower and upper of the tapered channel with increasing $\mathrm{m}$. the stream for different values of $\phi$ are shown in fig.(16), it is examined that the size of bolus decreasing rapidly in the lower and upper part of channel with increasing of $\phi$. Effect of a are shown in fig.(17), it is noticed that the size of bolus increasing in the lower and upper part of channel with increasing of $a$. fig(18) shows the effects of $b$ and it is observed that the bolus decrease in size with an increase in $b$. which is the same behavior of effect of $\mathrm{M}$ and plotted on fig.(19), figs.(20) and (21)shows the effects of $\mathrm{k}$ and $\theta$ respectively and it is observed that an increase in $\mathrm{k}$ and $\theta$ causes decreasing in size of bolus in the upper and lower part of channel

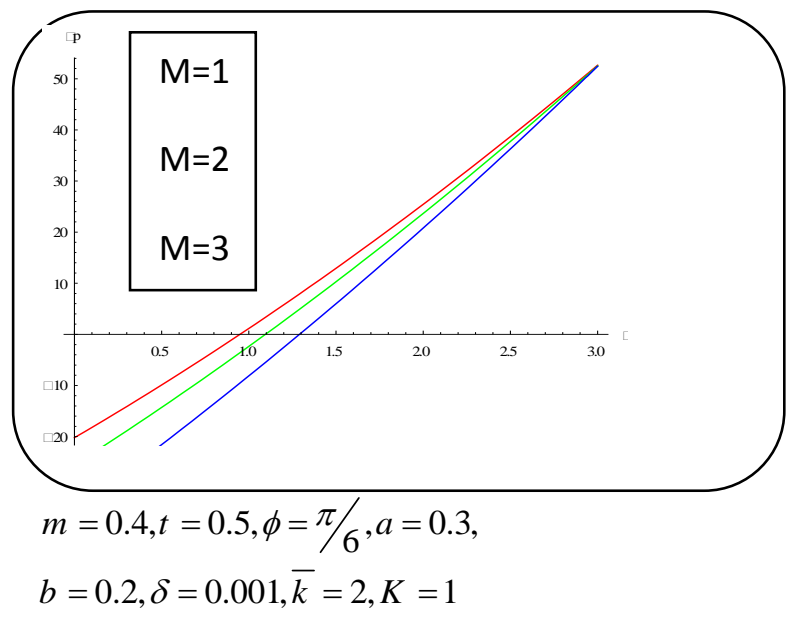

Fig.(2).Effect of Hartmann number M on $\Delta p$

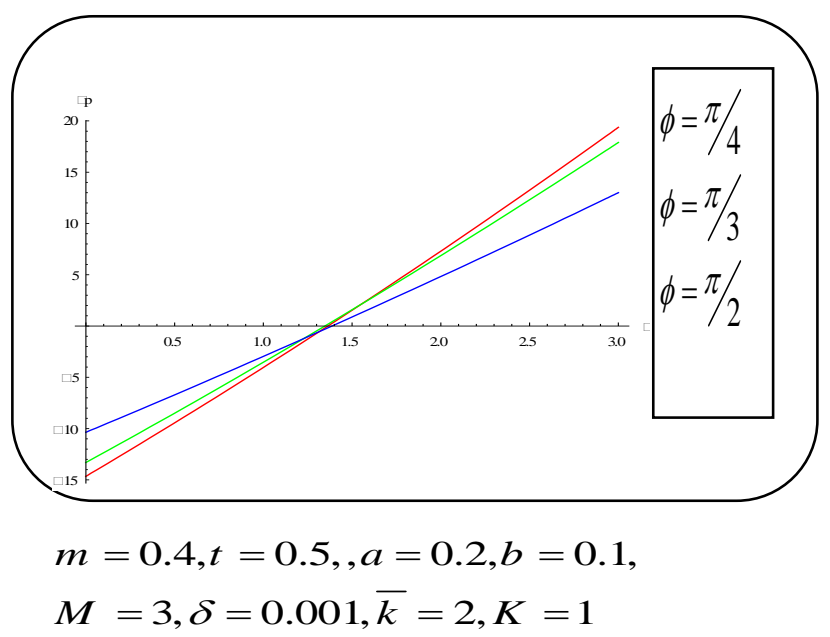

Fig.(3). Effect of phase difference $\phi$ on $\Delta p$ 


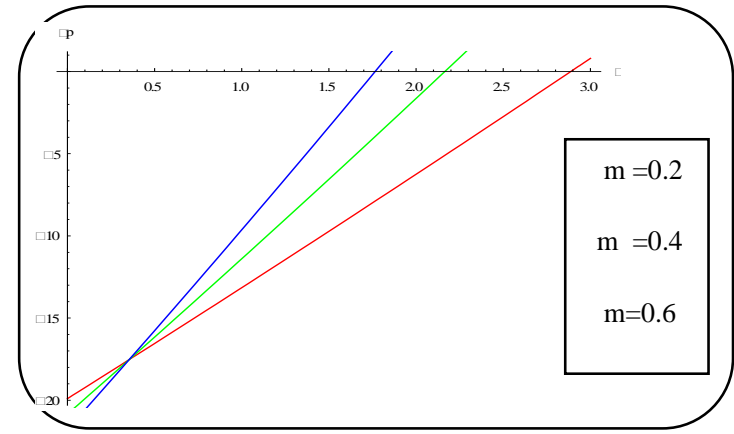

$t=0.5, \phi=\frac{\pi}{2}, a=0.2, b=0.1$,

$M=5, \delta=0.001, \bar{k}=2, K=1$

Fig.(4) Effect of non-uniform parameter m on $\Delta p$

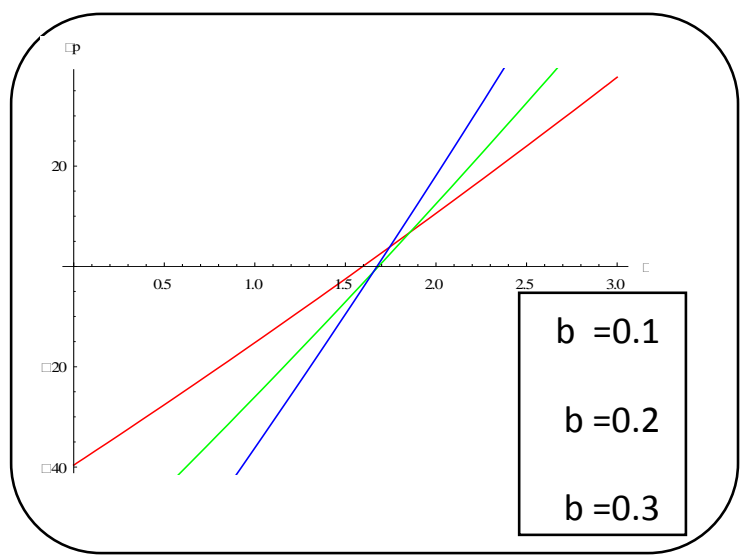

$m=0.4, t=0.5, \phi=\pi / 6, a=0.3$,

$M=5, \delta=0.001, \bar{k}=2, K=1$

Fig.(6 ) Effect of amplitude of upper wall of channel b on $\Delta p$

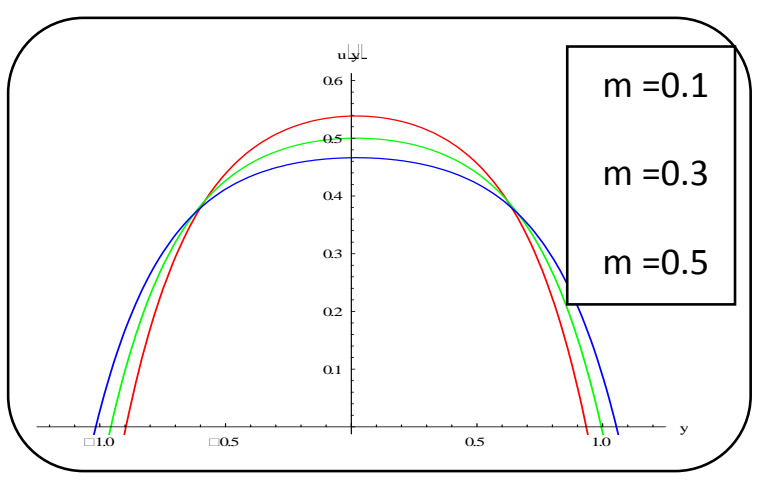

$t=0.5, \phi=\pi / 6, a=0.2, b=0.1, M=5$,

$\delta=0.0001, \bar{k}=2, K=1, \theta=1, x=0.3$

Fig.(8) Effect of non -uniform parameter m on axial velocity $\mathrm{u}(\mathrm{y})$
Journal of Advances in Mathematics

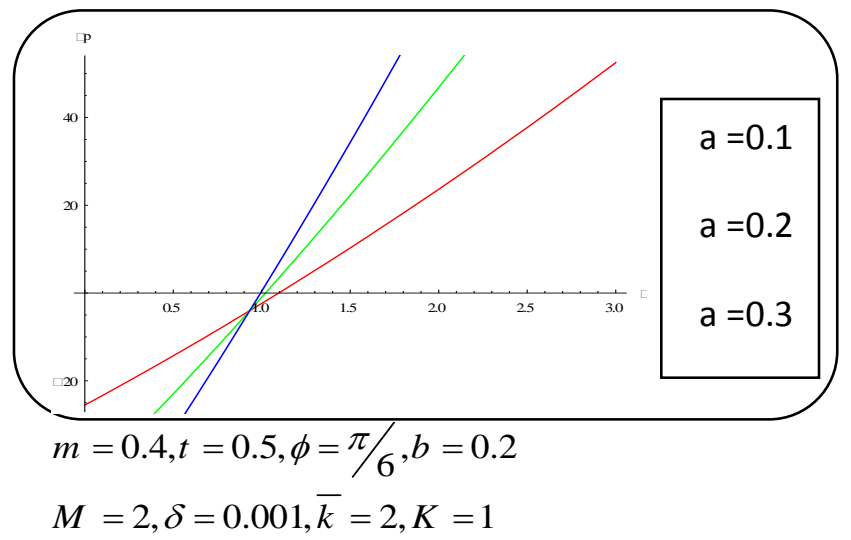

Fig.(5) Effect of the amplitude of lower wall of channel a on $\Delta p$

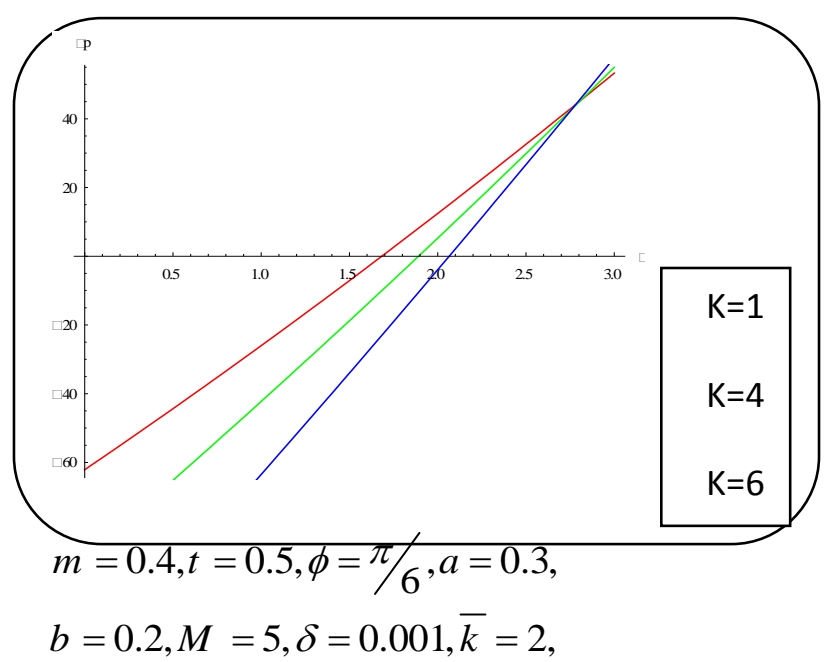

Fig.(7) Effect of the inverse of Dercy number K on $\Delta p$

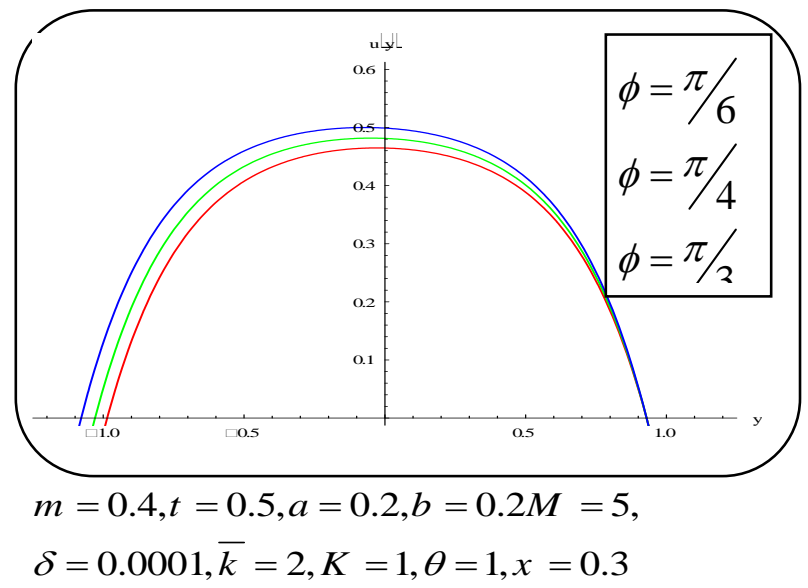

Fig.(9) Effect of phase difference on the axial velocity on $\mathrm{u}(\mathrm{y})$ 


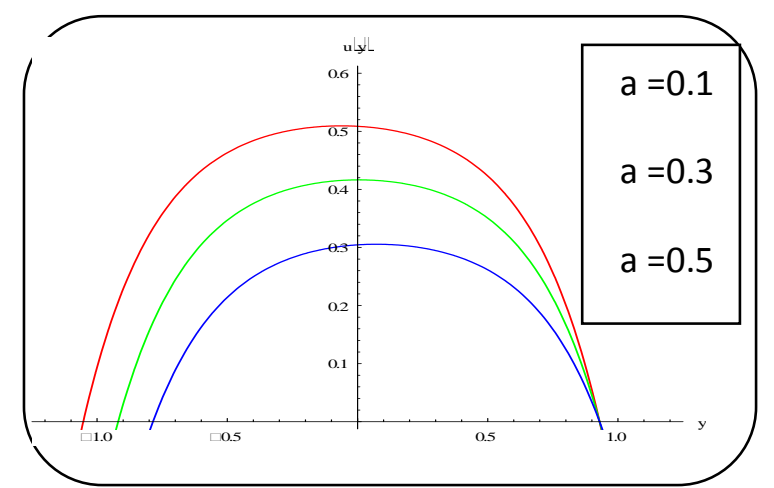

$m=0.4, t=0.5, \phi=\pi / 6, b=0.2 M=5$,

$\delta=0.0001, \bar{k}=2, K=1, \theta=1, x=0.3$

Fig(10) Effect of amplitude of lower wall of channel on axial velocity $\mathrm{u}(\mathrm{y})$

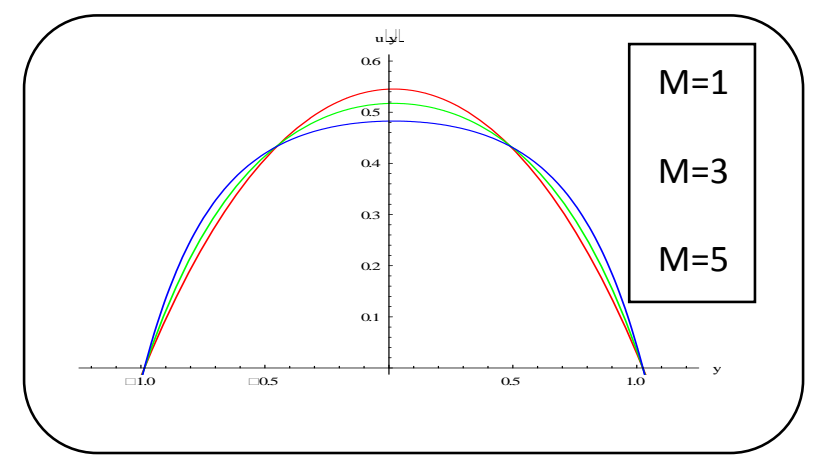

$m=0.4, t=0.5, \phi=\pi / 6, a=0.2, b=0.1$,

$\delta=0.0001, \bar{k}=2, K=1, \theta=1, x=0.3$

Fig.(12) Effect of Hartmann number M on axial velocity $\mathrm{u}(\mathrm{y})$

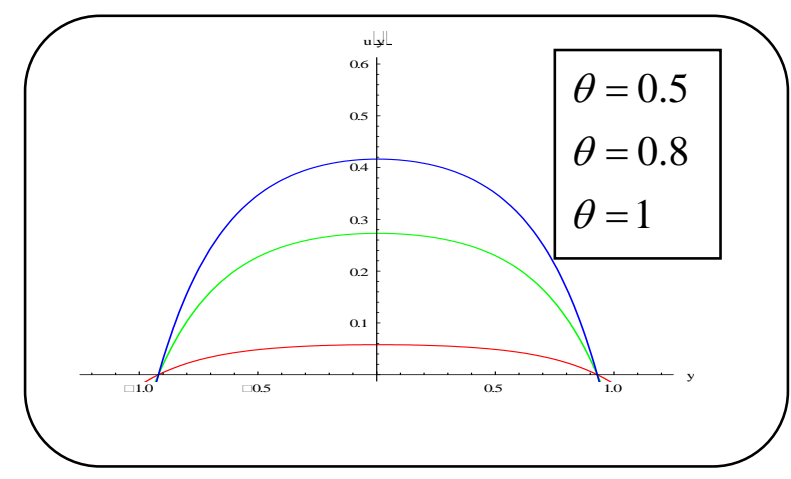

$m=0.4, t=0.5, \phi=\pi / 6, a=0.3, b=0.2$,

$M=5, \delta=0.0001, \bar{k}=2, K=1, x=0.3$

Fig.(14) Effect of the time average $\theta$ on axial velocity $\mathrm{u}(\mathrm{y})$

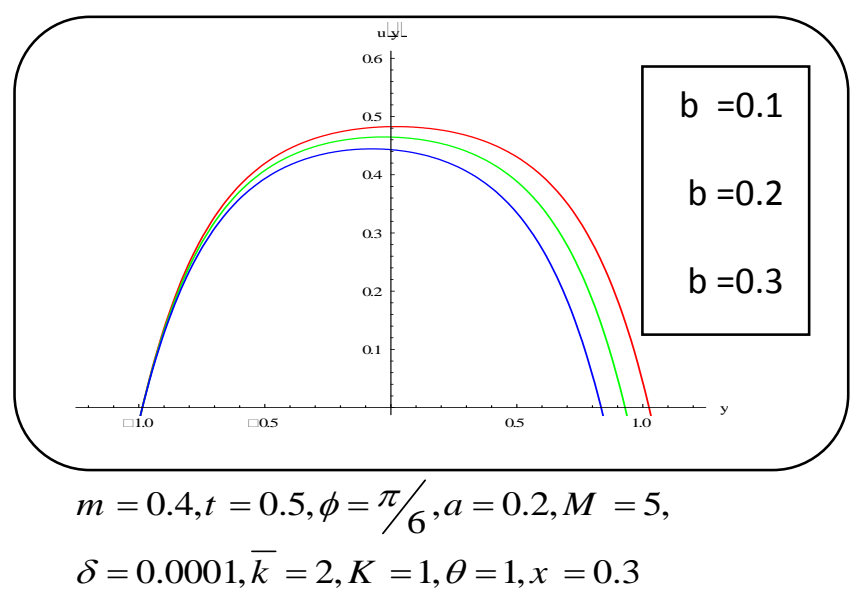

Fig.(11) Effect of amplitudes of upper wall channel on axial velocity $\mathrm{u}(\mathrm{y})$

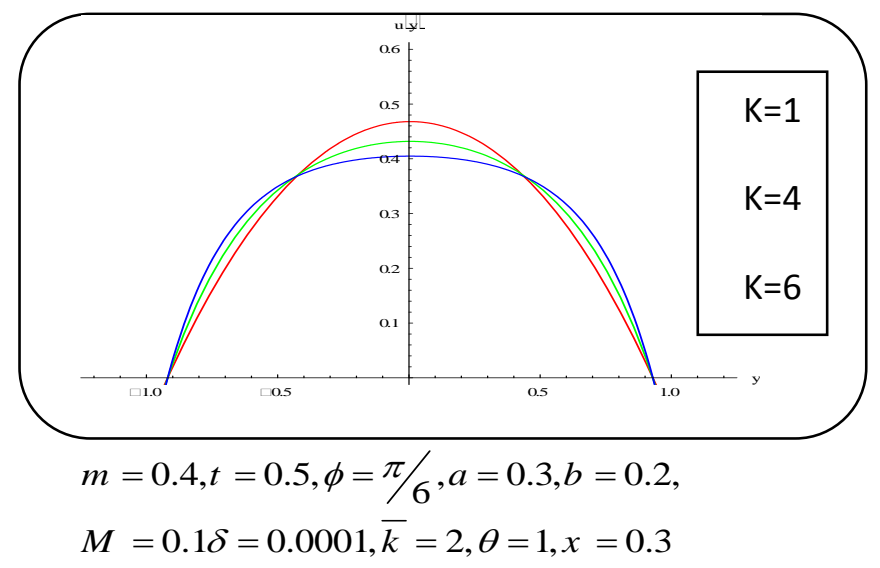

Fig(13) Effect of the inverse of the Dercy number K on axial velocity $\mathrm{u}(\mathrm{y})$. 

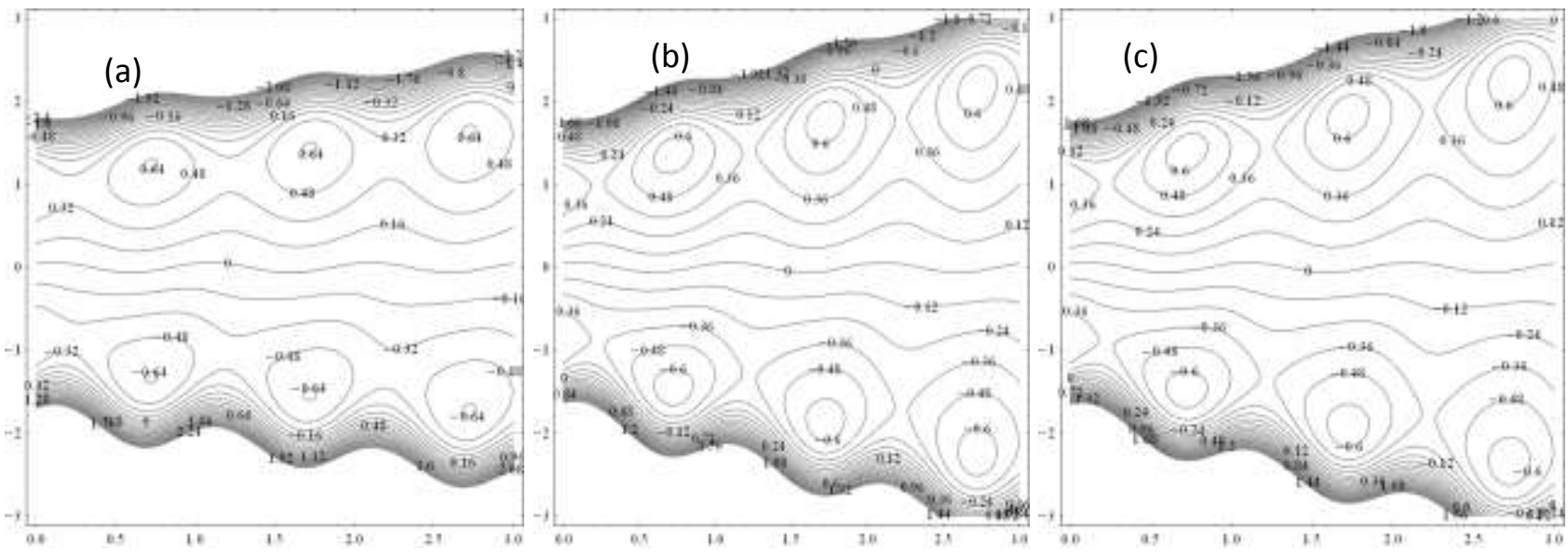

$t=0.5, \phi=\pi / 6, a=0.2, b=0.1, M=5, \delta=0.0001, \bar{k}=2, K=1, \theta=1$

(a) $m=0.2,(b) m=0.4,(c) m=0.44$

Fig.(15) Stream lines for
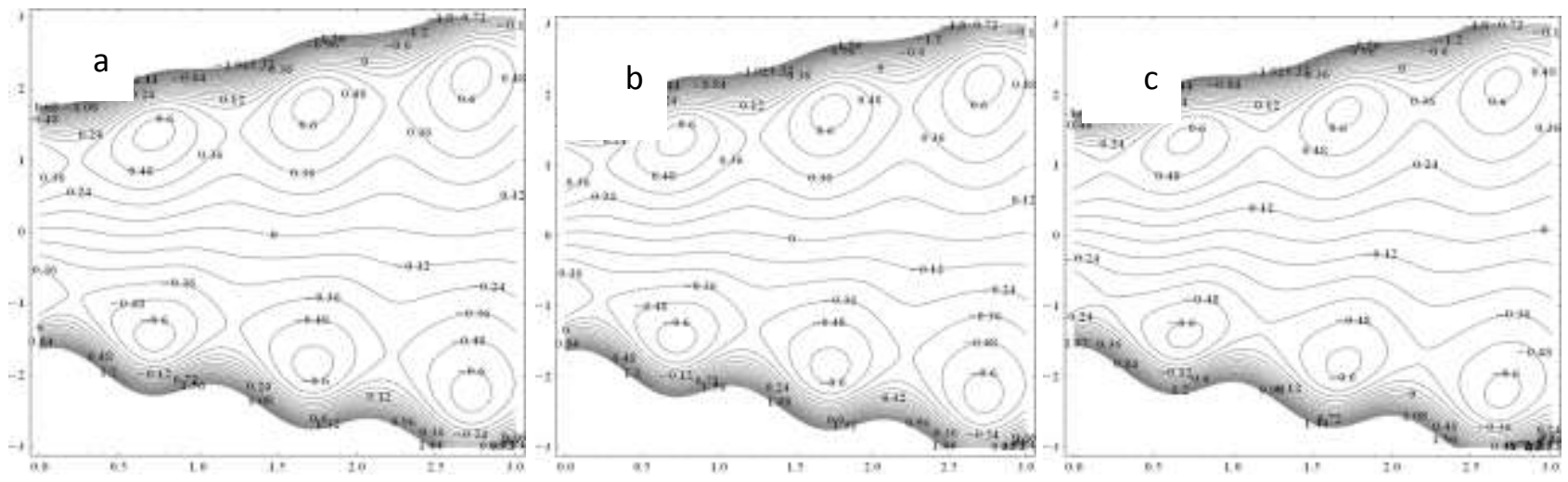

$m=0.4, t=0.5, a=0.2, b=0.1, M=5, \delta=0.0001, \bar{k}=2, K=1, \theta=1$

(a) $\phi=\pi / 6,(b) \phi=\pi / 4,(c) \phi=\pi / 3$

Fig.(16) Stream lines for
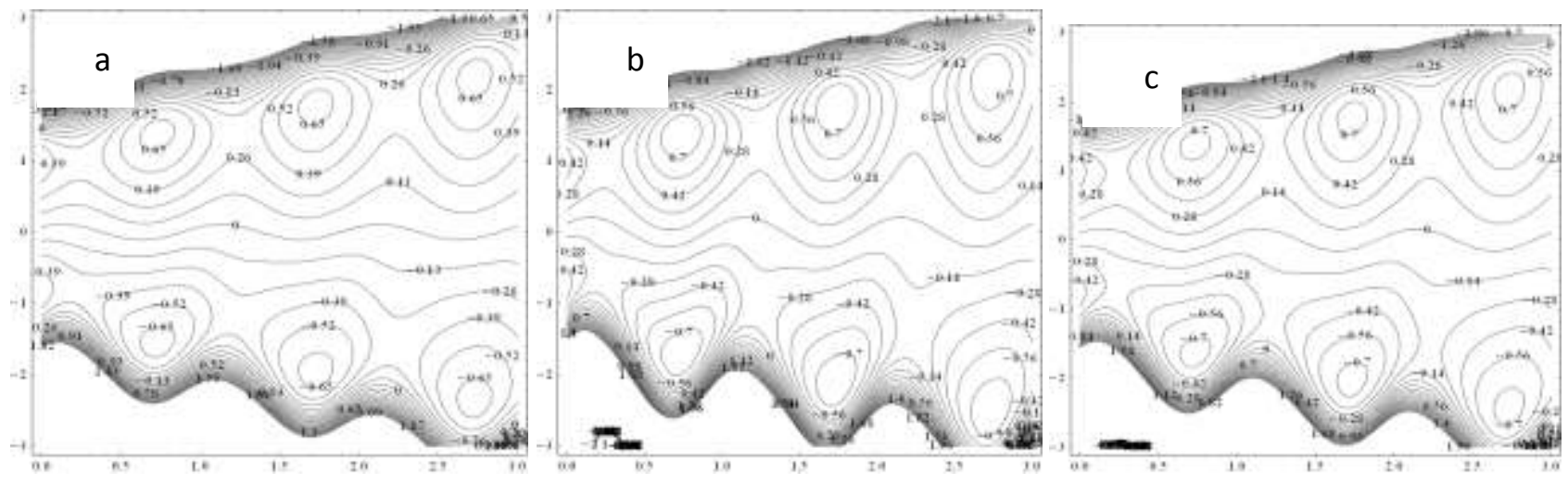

$$
\begin{aligned}
& m=0.4, t=0.5, \phi=\pi / 6, b=0.1, M=5, \delta=0.0001, \bar{k}=2, K=1, \theta=1 \\
& (a) a=0.3,(b) a=0.4,(c) a=0.5
\end{aligned}
$$

Fig.(17) Stream lines for 

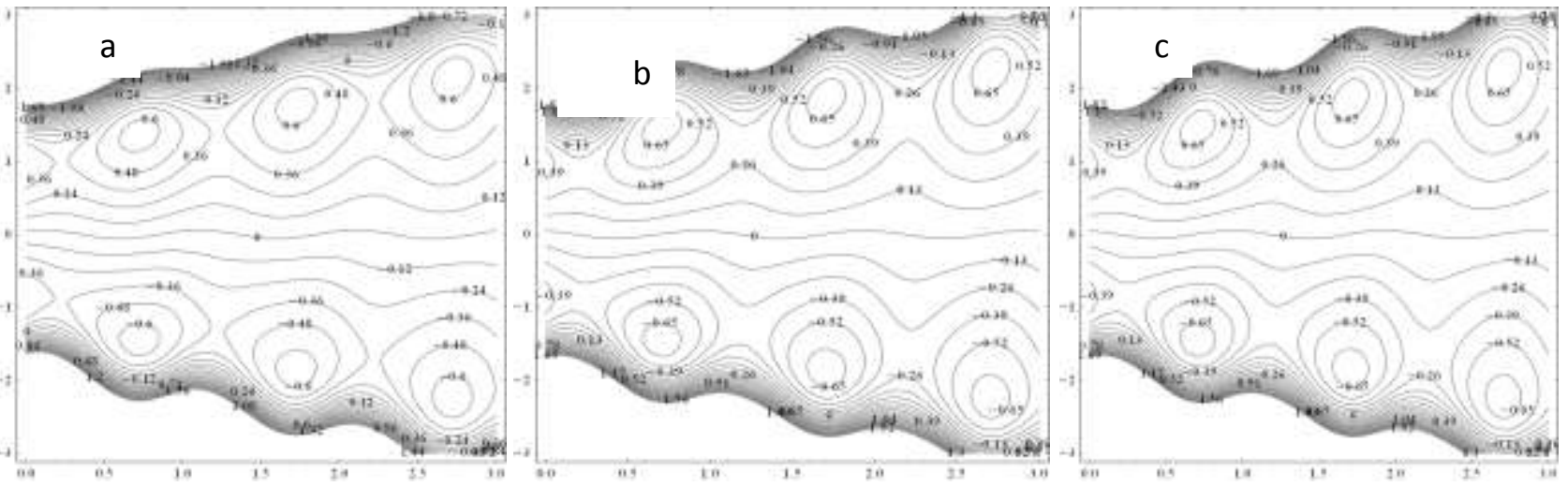

$$
\begin{aligned}
& m=0.4, t=0.5, \phi=\pi / 6, a=0.2, M=5, \delta=0.0001, \bar{k}=2, K=1, \theta=1 \\
& (a) b=0.4,(b) b=0.2,(c) b=0.22
\end{aligned}
$$

Fig.(18) Stream lines for
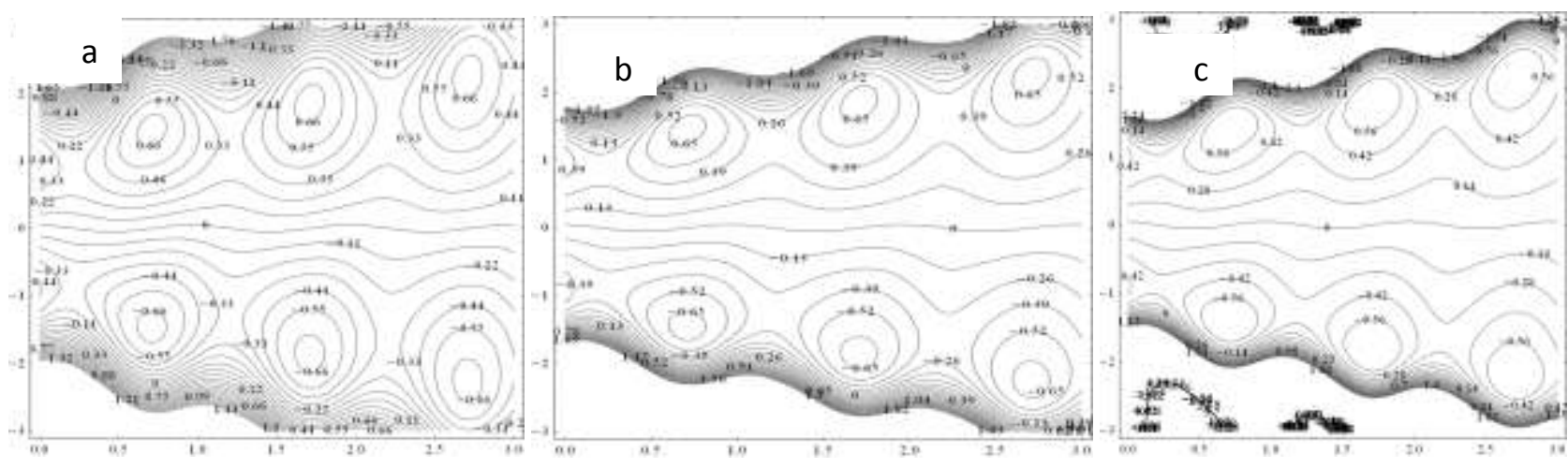

$$
\begin{aligned}
& m=0.4, t=0.5, \phi=\pi / 6, a=0.2, b=0.2, \delta=0.0001, \bar{k}=2, K=1, \theta=1 \\
& (a) M=2,(b) M=5,(c) M=8
\end{aligned}
$$

Fig.(19) Stream lines for
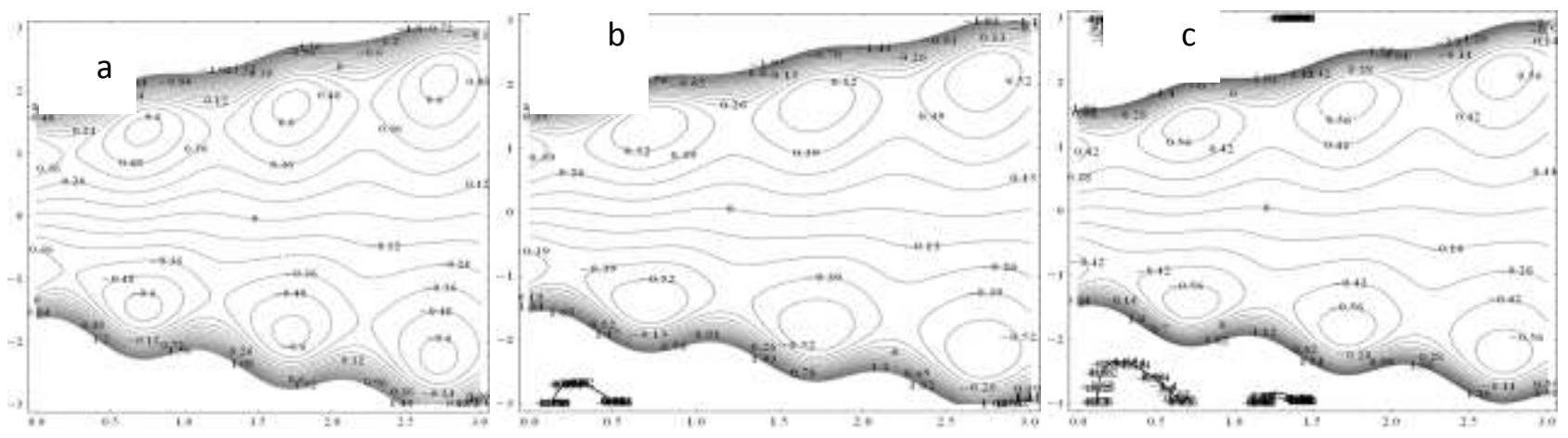

$m=0.4, t=0.5, \phi=\pi / 6, a=0.2, b=0.1, M=5, \delta=0.0001, \bar{k}=2, \theta=1$

(a) $K=1,(b) K=4,(c) K=6$

Fig.(20) Stream lines for 

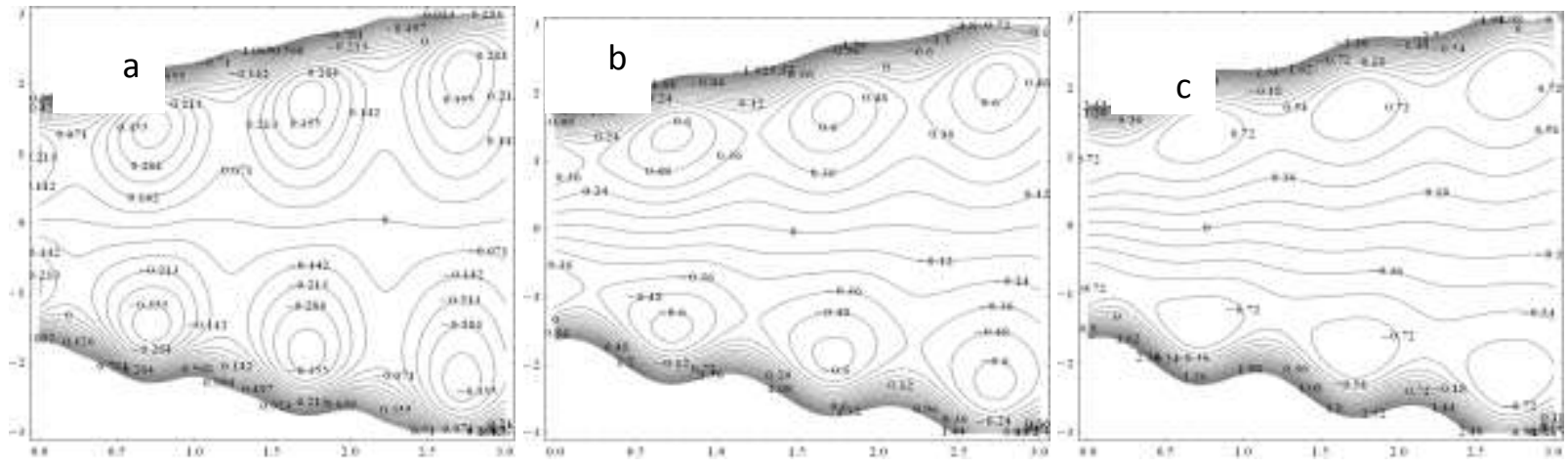

$$
\begin{aligned}
& m=0.4, t=0.5, \phi=\pi / 6, a=0.2, b=0.1, M=5, \delta=0.0001, \bar{k}=2, K=1 \\
& (a) \theta=0.5,(b) \theta=1,(c) \theta=1.5
\end{aligned}
$$

Fig.(21) Stream lines for
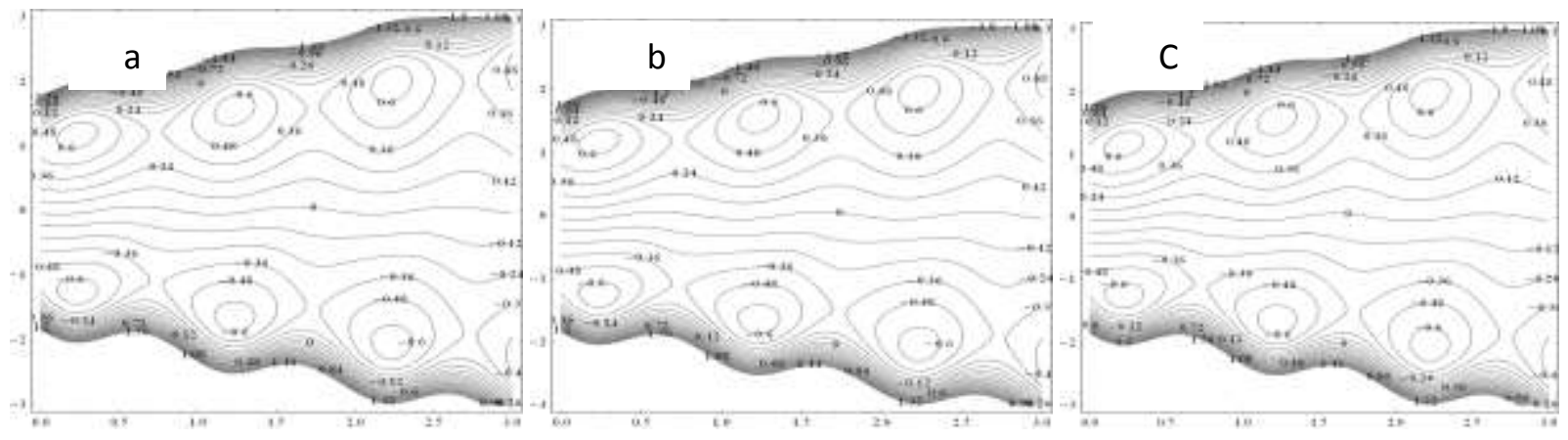

$$
\begin{aligned}
& m=0.4, \phi=\pi / 6, a=0.2, b=0.1, M=5, \delta=0.0001, \bar{k}=2, K=1, \theta=1 \\
& (a) t=0.01,(b) t=0.5,(c) t=1
\end{aligned}
$$

Fig.(22) Stream lines for

\section{CONCLUDING REMARKS}

In this paper, we investigated the peristaltic transport of Walters -B fluid through aporous medium in the tapered a symmetric channel under the influence of magnetic field, the channel a symmetry is produced by choosing the peristaltic waves train on the non- uniform walls to have different amplitudes and phases along-wave length and low Reynolds number approximations are adopted. A regular perturbation method is employed to obtain the expression for stream function, axial velocity and pressure gradient- numerical study has been conducted for average rise in pressure over a wave length. The effects of Hartmann number (M), the inverse of Darey number (k), wave amplitudes (a \&b), channel width $(\mathrm{m})$ and phase angle $\phi$ on the pressure rise, axial velocity and stream lines are also investigated in detail. It found that : 
1. The pressure rise over a wave length $\Delta p$ increase with an increase in $\mathrm{m}$, a in the pumping $\Delta p>0$ while the situation is reversed in the free pumping $\Delta p=0$ and Co-pumping $\Delta p<0$

2. The pressure rise over a wave length $\Delta p$ increase with an increase in $\mathrm{b}, \mathrm{k}$ in the pumping $\Delta p>0$ and free pumping $\Delta p=o$ while the situation is reversed in the Co-pumping $\Delta p<0$

3. The pressure rise over a wave length $\Delta p$ increase with an increase in $\mathrm{M}$ in the pumping $\Delta p=0$ while the situation is conversely in the pumping $\Delta p>0$ and Co-pumping $\Delta p<0$

4. the pressure rise over a wave length $\Delta p$ increase with an increase in $\phi$ in the Co-pumping $\Delta p<0$ and its conversely in the pumping $\Delta p>0$

5. the axial velocity $u$ decreasing with an increase of $m, b, k$ and $M$.

6. the axial velocity $\mathrm{u}$ increasing with an increase of $\phi$, a and $\theta$.

7. The size of the trapped bolus increase with an increase of $m$ and a, while decrease with an increase of $\phi, b, M, \theta$ and $\mathrm{k}$.

\section{REFERENCES}

1. Akram, S., Nadeem, S.,(2013), Influence of induced magnetic field and heat transfer on the peristaltic motion of a Jeffrey fluid in an a symmetric channel closed form solutions. J. Magn.Magn.Mater. 328, 11-20.

2. Burns, J.C. , Pareks, J. , (1967), peristaltic motion. J. Fluid Mech. 29, 731-743.

3. Fung, . Y. C. ,Yih, c. S. , (1968), Peristaltic transport. J. App. Mech.35, 669-675.

4. Gad, N. S. , (2014), Effects of hall currents on peristaltic transport with compliant walls. Appl. Math. Comput. 235, 546554.

5. Hayat, T. , Javed, M. , Ali, N. , (2008), MHD Peristaltic transport of Jeffery fluid in a channel with compliant walls and porous space. Transp. Porous Med. 74, 259-274.

6. Hayat, T. , Javed, M. , Asghar, S. , (2008), MHD peristaltic motion of Johnson-Segalman fluid in a channel with compliant walls. Phys. Lett. A. 372, 5026-5036.

7. Hina, S. , Mustafa, M. , Hayat, T. , Alotaibi, N.D. , (2015), on peristaltic motion of Pseudo plastic fluid in a curved channel with heat / mass transfer and wall properties, Appl. Math comput. 263, 378-391.

8. Hina, S. , (2016) MHD Peristaltic transport of Eyring- Powell fluid with heat / mass transfer, wall properties and slip conditions, J. Magn. Magn, Mater. 404,148-158.

9. Javed, M. , Hayat, T. , Alsaedi, A. , (2014) Peristaltic flow of Burgers fluid with compliant walls and heat transfer Appl. Math. Comput. 244, 654-671.

10. Kothandapani, M. , prakash, J. (2015), Effects of thermal radiation and chemical reactions on peristaltic flow of a Newtonian nano fluid under inclined magnetic field in a generalized vertical channel using homotopy perturbation method, Asia- Pacif. J. Chem. Eng. 10, 259-272.

11. Kothandapani, M. , prakash, J. (2015), Effects of thermal radiation parameter and magnetic field on the peristaltic motion of Williamson nano fluid in a tapered a symmetric channel. Int. J. Heat Mass trans. 81, 234-245. 
12. Kothandapani, M. , prakash, J., Pushparaj, V. , (2015), Effects of Heat transfer, magnetic field and space porosity on peristaltic flow of a Newtonian fluid

13. Latham, T. W. , (1966), fluid motion in peristaltic pump (M. S. Thesis). MIII, Cambridge, Mass.

14. Mekheimer, Kh. S. , (2003), Non-linear peristaltic transport of magneto-hydrodynamic flow in an inclined planar channel. Arab. J. Sci. Eng. 28, 183-201.

15. Mekheimer, Kh. S. , Al-Arabi, T. H. , (2003), Non- linear peristaltic transport of MHD flow through a porous medium. IJMMS26, 1663-1682.

16. Mekheimer, Kh. S. , Elkot, M. A. ,(2008), The micro polar fluid model for blood flow through a tapered artery with a stenos is, Acta Mech. Sin, 24, 673-644.

17. Muthu, P. , Kumar, B. V. R. , Chandra, P. , (2008), Peristaltic motion of micro polar fluid in circular cylindrical tubes, Effect of wall properties, Appl. Math. Model. 32, 2019-2033.

18. Javed, M. , Hayat, T. , Mustafa, M. , Ahmed, B. , (2016), Velocity and thermal slip effects on peristaltic motion of walters-B fluid, Internation. Journal of heat and mass transfer. 96, 210-217.

19. Nadeem, S. , Akbar, N. S. , (2010), Peristaltic flow of sisko fluid in a uniform inclined tube. Acta Mech. Sin. 26, 675683.

20. Radhakrish namacharya, G. , Srinivasulu, C. , (2007), Influence of wall properties on peristaltic transport with heat transfer, C. R. Mec. 335,369-373.

21. Riaz, A. , Nadeem, S. , Ellahi, R. , Akbar, N. S. , (2014), The influence of wall flexibility on unsteady peristaltic flow of prandtl fluid in a three dimensional rectangular duct, Appl. Math. Comput . 241, 389-400.

22. Shapiro, A. H. , Jaffrin, M.Y., Weinberg, S. L. , (1969), peristaltic pumping with long wave lengths at low Reynolds number. J. Fluid Mech. 37, 799-825.

23. Srinivas, S., Kothandapani, M. , (2009), the influence of heat and mass transfer on MHD peristaltic flow through a porous space with compliant walls, Appl. Math. Comput. 213, 197-208.

24. srivasteva, L. M. , Srivastiva, V. P., Sinha, S. N. , (1983), Peristaltic transport of a physiological fluid part - K flow in non geometry-Biorheology 20, 153-166.

25. Takabatake, S. , Ayukawa, K. , (1982), Numerical study of two - dimensional Sional peristaltic flows J. fluid Mech. 12 , 439-456.

26. Walters, K. , (1962), Non- Newtonian effects in some elastic-viscous liquids whose behavior linear equations of state. Quart. J. Mech. Appl. Math. 15, 63-76. 